\begin{tabular}{|c|l|}
\hline Title & Proposal and analysis of a ferromagnetic triple barrier resonant-tunneling spin filter \\
\hline Author(s) & Uemura, T.; Marukame, T.; Y amamoto, M. \\
\hline Citation & $\begin{array}{l}\text { IEEE Transactions on Magnetics, 39(5), 2809-2811 } \\
\text { https://doi.org/10.1109/1MA G.2003.815719 }\end{array}$ \\
\hline Issue Date & 2003-09 \\
\hline Doc URL & http://hdl.handle.net/2115/5578 \\
\hline Rights & Copyright $\odot 2003$ A merican Institute of Physics \\
\hline Type & article \\
\hline File Information & ITM39-5.pdf \\
\hline
\end{tabular}

Instructions for use 


\title{
Proposal and Analysis of a Ferromagnetic Triple-Barrier Resonant-Tunneling Spin Filter
}

\author{
Tetsuya Uemura, Takao Marukame, and Masafumi Yamamoto, Member, IEEE
}

\begin{abstract}
A novel spin filter consisting of a triple-barrier resonant tunneling system in the form $F / I / N / I / F / I / F$ is proposed, where $F, I$, and $N$ represent a ferromagnetic material, an insulator, and a nonmagnetic material, respectively. The spin-dependent tunneling current in the triple-barrier resonant tunneling system is calculated theoretically on the basis of a Tsu-Esaki formula to investigate the output tunnel current polarization. Detailed calculations using the GaMnAs/AlAs/GaAs material system show that the two clear split peaks originating from up- and down-spin holes appear in the current-voltage $(I-V)$ curve due to spin splitting of the energy levels formed in the ferromagnetic quantum well. The polarization can reach more than $98 \%$ at the peak positions in the $I-V$ curve.
\end{abstract}

Index Terms-GaMnAs, resonant tunneling, spin filter, spin splitting.

\section{INTRODUCTION}

$\mathbf{R}$ ESONANT tunneling involving electron spin is attracting much attention because it will enable developing new functional spin-dependent devices in future electronics [1]-[7]. A spin filter is one such key device. Magnetic quantum well (QW) structures have been considered applicable as spin filters because the resonant levels formed in a QW are split depending on the spin states due to the exchange interaction [3], [5]. The output current, however, does not show clear peak splitting due to the overlapping of the resonant conditions. In this paper, we propose a new type of spin filter that uses a triple-barrier structure. The proposed device consists of a nonmagnetic and a ferromagnetic QW. The inherent peaked current-voltage $(I-V)$ characteristics in the triple-barrier resonant structure result in a distinct peak separation of the up-spin and the down-spin components regarding the bias voltage, which leads to an exceedingly high spin polarization. We theoretically calculated the spin-dependent tunneling current and the tunnel-barrier-width dependence of both the spin polarization and the current density.

\section{TRIPLE-BARRIER SPIN FILTER}

Consider first the double-barrier magnetic QW structure shown in the inset of Fig. 1. The well and emitter regions

Manuscript received January 2, 2003. This work was supported in part by a Grant-in-Aid for Scientific Research from the Ministry of Education, Culture, Sports, Science and Technology, Japan.

The authors are with the Division of Electronics and Information Engineering, Hokkaido University, Sapporo 060-8628, Japan (e-mail: uemura@ nano.eng.hokudai.ac.jp).

Digital Object Identifier 10.1109/TMAG.2003.815719

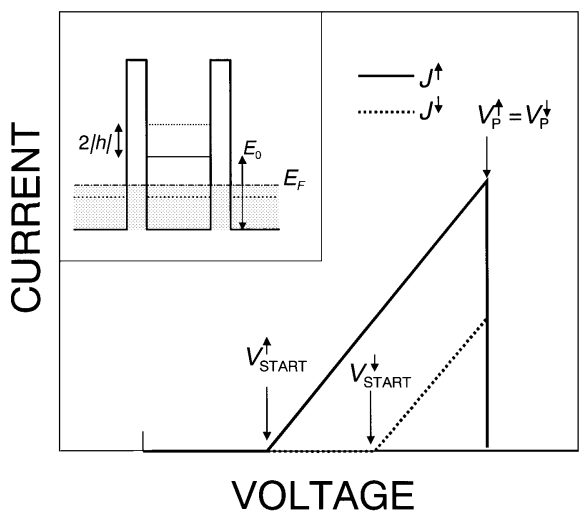

Fig. 1. Schematic $I-V$ characteristics of double-barrier QW at $0 \mathrm{~K}$. The $J^{\uparrow}$ and $J \downarrow$ cannot be split from each other because $V_{P}^{\uparrow}=V_{P}^{\downarrow}$.

consist of a ferromagnetic material. The Schrödinger equation may be written as

$$
\left(-\frac{\hbar^{2}}{2 m} \nabla^{2}+U-\boldsymbol{h} \cdot \boldsymbol{\sigma}\right) \Psi(\boldsymbol{r})=E \Psi(\boldsymbol{r})
$$

where $U$ is potential energy, $\boldsymbol{h}$ is the molecular field, and $\sigma(=2 s)$ is the conventional Pauli spin operator. Due to the internal exchange energy $\boldsymbol{- h} \cdot \boldsymbol{\sigma}$, the resonant level in the $\mathrm{QW}$ is split by $2|\boldsymbol{h}|$, depending on the spin states. Because the resonant tunneling starts when the Fermi energy in the emitter $\left(E_{F}\right)$ is aligned to the energy level formed in the QW, the voltages at which the resonant tunneling current start to flow are given by

$$
\begin{aligned}
& V_{\text {start }}^{\uparrow}=2\left(E_{0}-|\boldsymbol{h}|-E_{F}\right) / q \quad \text { for up-spin } \\
& V_{\text {start }}^{\downarrow}=2\left(E_{0}+|\boldsymbol{h}|-E_{F}\right) / q \quad \text { for down-spin }
\end{aligned}
$$

where $E_{0}$ represents the energy difference between the resonant level and energy band edge of the QW. The resonant tunneling current increases as the applied voltage increases until the energy band edge of the emitter is aligned to the resonant level in the QW. Because the band edge of the emitter is also split depending on the spin state, the peak voltages at which the resonant tunneling current becomes maximum are equal for up-spin and down-spin electrons, and are given by

$$
V_{P}^{\uparrow}=V_{P}^{\downarrow}=2 E_{0} / q
$$

Here, we assume that all the magnetization directions in each ferromagnet are parallel. Fig. 1 shows schematic $I-V$ characteristics of the double-barrier magnetic $\mathrm{QW}$ structure under the zero-temperature limit. Because the $V_{P}^{\uparrow}$ is equal to $V_{P}^{\downarrow}$, the 


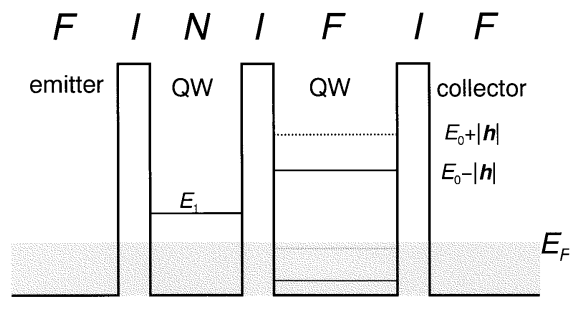

Fig. 2. Energy band profile of the proposed triple-barrier structure. A nonmagnetic $\mathrm{QW}$ is inserted between the ferromagnetic emitter and ferromagnetic QW.

up-spin current $\left(J^{\uparrow}\right)$ and the down-spin current $\left(J^{\downarrow}\right)$ cannot be split from each other.

In order to split the $J^{\uparrow}$ and $J \downarrow$ components, we propose a new structure in which a nonmagnetic $\mathrm{QW}$ is inserted between the ferromagnetic emitter and the ferromagnetic QW. Fig. 2 shows an energy-band profile of the proposed triple-barrier resonanttunneling structure. In this triple-barrier structure, the resonant tunneling occurs only when the energy levels formed in the two wells are aligned with each other. In other words, the $I-V$ curve exhibits a shape like a delta-function. The $J^{\uparrow}$ and $J^{\downarrow}$, therefore, can be split by $6|\boldsymbol{h}| / q$, because the peak voltages of the $J^{\uparrow}$ and $J \downarrow$ are given by

$$
\begin{aligned}
& V_{P}^{\uparrow}=3\left(E_{0}-|\boldsymbol{h}|-E_{1}\right) / q \quad \text { for up-spin } \\
& V_{P}^{\downarrow}=3\left(E_{0}+|\boldsymbol{h}|-E_{1}\right) / q \quad \text { for down-spin }
\end{aligned}
$$

where $E_{1}$ is the energy level formed in the nonmagnetic QW.

\section{ANALYSIS}

The $J^{\uparrow}$ and $J \downarrow$ of the triple-barrier QW structure were calculated using a Tsu-Esaki formula [9] for a GaMnAs/AlAs/GaAs material system. We assumed the ballistic or quantum-coherent transport condition. The triple-barrier QW structure consists of a GaMnAs emitter, a 3.1-nm-thick AlAs barrier, a 4.2-nm-thick GaAs well, a 3.1-nm-thick AlAs barrier, a 9.0-nm-thick GaMnAs well, a 3.1-nm-thick AlAs barrier, and a GaMnAs collector. We assumed that both the GaMnAs and the GaAs layers were p-type with 100-meV Fermi energies and that the AlAs was undoped. We also assumed that the band parameters of GaMnAs were the same as those of GaAs, because the band structure of GaMnAs is very similar to that of GaAs. We used the band discontinuity between GaMnAs and AlAs of $0.5 \mathrm{eV}$ and the spin splitting energy $(2|\boldsymbol{h}|)$ of $44 \mathrm{meV}$. We designed the device structures such that the energy level formed in the GaAs QW $\left(E_{1}\right)$ lies between the Fermi energy of the emitter and the first excited state of the up-spin carrier $\left(E_{0}-|\boldsymbol{h}|\right)$.

The $J^{\uparrow}$ and $J^{\downarrow}$ are given by

$$
\begin{aligned}
J^{\uparrow, \downarrow}\left(V_{a}\right)=\sum_{j} & \frac{q m_{j} k_{B} T}{4 \pi^{2} \hbar^{3}}\left[\int_{-|\boldsymbol{h}| \sigma}^{\infty} \mathcal{T}^{\uparrow, \downarrow}\left(E_{z}\right)\right. \\
& \left.\times \ln \frac{1+\exp \left(\frac{E_{F}+|\boldsymbol{h}| \sigma-E_{z}}{k_{B} T}\right)}{1+\exp \left(\frac{E_{F}+|\boldsymbol{h}| \sigma-E_{z}-q V_{a}}{k_{B} T}\right)} d E_{z}\right]
\end{aligned}
$$

where $\sigma$ takes a value of +1 for up-spin and -1 for down-spin, $V_{a}$ is the applied bias voltage, $m_{j}$ is the hole mass, $k_{B}$ is the

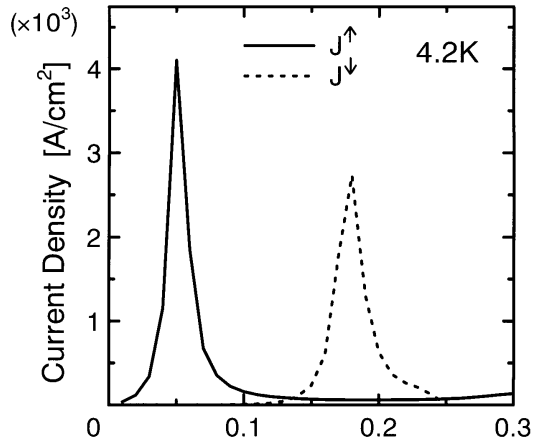

(a)

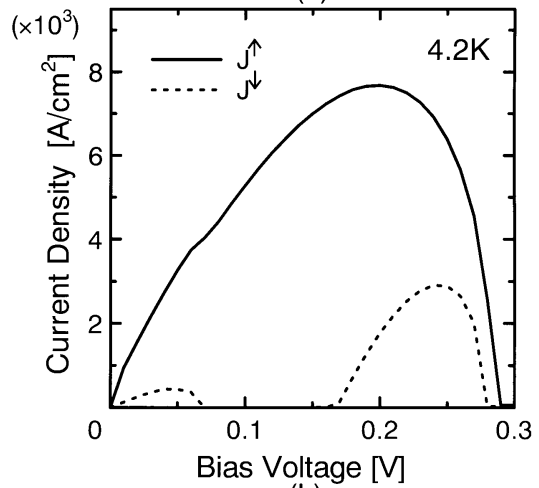

(b)

Fig. 3. Calculated $I-V$ characteristics of magnetic QW structures. (a) Triple barrier. (b) Double barrier.

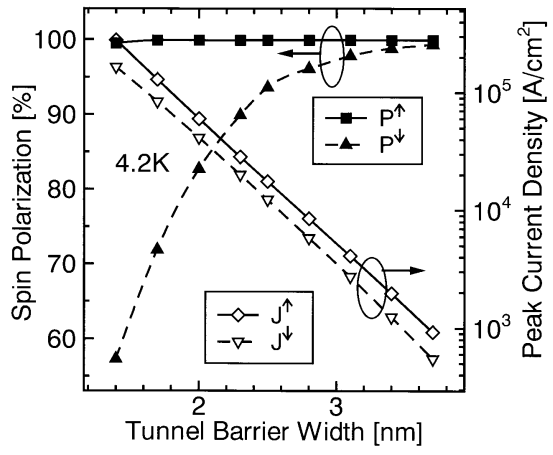

Fig. 4. Tunnel-barrier-width dependence of polarization and current density.

Boltzmann constant, $T$ is the temperature, and $\mathcal{T}^{\uparrow, \downarrow}\left(E_{z}\right)$ is the transmission probability of a hole having the longitudinal energy of $E_{z}$. We set the origin of the potential energy to the valence band edge of the GaAs well. The summation in (5) was done over the light hole $\left(m_{j}=0.08\right)$ and the heavy hole $\left(m_{j}=0.45\right)$ distributed in the emitter. The transmission probability was calculated by using the transfer matrix method at every bias point.

\section{RESULTS AND DISCUSSION}

Fig. 3(a) shows the calculated $I-V$ characteristics of the proposed triple-barrier structure consisting of a nonmagnetic GaAs QW and a magnetic GaMnAs one. Fig. 3(b) shows those of the double-barrier structure consisting of a GaMnAs QW for comparison. The temperature was $4.2 \mathrm{~K}$. In the double-barrier structure, the $J^{\uparrow}$ and $J \downarrow$ do not split from each other because the resonant conditions for up-spin and down-spin holes are over- 
lapped as shown in Fig. 1. In the triple-barrier structure, on the other hand, the $J^{\uparrow}$ and $J \downarrow$ split from each other. The GaAs QW works as an energy filter, which enables reducing of the energy width of injected holes to the GaMnAs QW.

Fig. 4 shows the tunnel-barrier-width dependence of the polarization and the current density at each peak voltage. The current density decreases exponentially with increasing barrier width, while the polarizations increase monotonically and exceed $98 \%$ at a 3.4-nm barrier width. The increase in polarization is caused by the narrowing of the width of resonant levels formed in the QWs with increasing tunnel-barrier width. Moreover, almost no decrease in the up-spin polarization was observed, even in the region where the barrier is thin.

\section{CONCLUSION}

We proposed a new triple-barrier resonant-tunneling spin filter consisting of a nonmagnetic and a magnetic QW. Detailed calculations using a GaAs/AlAs/GaMnAs material system showed that it can produce high spin-polarized currents with polarizations exceeding $98 \%$.

\section{REFERENCES}

[1] A. Vedyayev, N. Ryzhanova, C. Lacroix, L. Giacomoni, and B. Dieny, "Resonance in tunneling through magnetic valve tunnel junctions," Europhys. Lett., vol. 39, pp. 219-224, 1997.

[2] X. Zhan, B. Li, G. Sun, and F. Pu, "Spin-polarized tunneling and magnetoresistance in ferromagnet/insulator(semiconductor) single and double tunnel junctions subjected to an electric field," Phys. Rev. B, vol. 56, pp. 5484-5488, 1997.

[3] T. Hayashi, M. Tanaka, and A. Asamitsu, "Tunneling magnetoresistance of a GaMnAs-based double barrier ferromagnetic tunnel junction," $J$. Appl. Phys., vol. 87, pp. 4673-4675, 2000.

[4] A. Voskoboynikov, S. S. Lin, C. P. Lee, and O. Tretyak, "Spin-polarized electronic current in resonant tunneling heterostructures," J. Appl. Phys., vol. 87 , pp. 387-391, 2000.

[5] J. C. Egues, C. Gould, G. Richter, and L. W. Molenkamp, "Spin filtering and magnetoresistance in ballistic tunnel junctions," Phys. Rev. B, vol. 64, pp. 195319_1-195319_5, 2001.

[6] T. Koga, J. Nitta, H. Takayanagi, and S. Datta, "Spin-filter device based on the Rashba effect using a nonmagnetic resonant tunneling diode," Phys. Rev. Lett., vol. 88, pp. 126601_1-126601_4, 2002.

[7] S. Yuasa, T. Nagahara, and Y. Suzuki, "Spin-polarized resonant tunneling in magnetic tunnel junctions," Science, vol. 297, pp. 234-237, 2002.

[8] H. Ohno, N. Akiba, F. Matsukura, A. Shen, K. Ohtani, and Y. Ohno, "Spontaneous splitting of ferromagnetic (Ga, Mn)As valence band observed by resonant tunneling spectroscopy," Appl. Phys. Lett., vol. 73, pp. 363-365, 1998.

[9] R. Tsu and L. Esaki, "Tunneling in a finite superlattice," Appl. Phys. Lett., vol. 22, pp. 562-564, 1973. 